\title{
An introductory to the special issue of International e- Conference on Integrated Bioinformatics and Computational Biology (ICIBCoB)
}

$\mathrm{E}$ ver-evolving nature has kept mankind on its toes to understand the intricacies associated with it; be it the mysteries associated with space research or biology. Over the years several advancements have taken place in the field of biotechnology such as the elucidation of the structure of DNA, sequencing strategies and Mass Spectrometry among many others. Historical improvements in the DNA sequencing methods made us realize the completion of human genome project in 2003, which gifted us with 3.2 billion bases of data to be annotated into meaningful information.

Our planet is habituated not only by humans but all other diverse species about which knowledge is very little or incomplete. Next generation and third generation sequencing approaches have certainly helped us in generating large volumes of different genomic and transcriptomic data. Relatively, there is an ocean of data available in different databases and their sizes are increasing exponentially with time. There is a wide gap between the size of the data and the annotations being made so far.

Deciphering the hidden information in the data require highly sophisticated skills of a bioinformatician and a computational biologist. It provides bioinformaticians an opportunity to unravel the mysteries of such complex biological systems by applying highly efficient and application-oriented bioinformatics field. It involves parallel processing of high throughput data and at the same time a multidisciplinary approach towards the problem solving. Thus far, computer-based concepts such as neural networks, support vector machines, Hidden Markov Models, Dynamic programming are being applied to solve various intricate tasks in bioinformatics.

Bioinformatics has become one of the indispensable parts of the biological research. It enables a biological scientist to think, relate and realize a biological problem in a multi-dimensional approach. Therefore, an integrated approach involving bioinformatics can lead us to discoveries from across the spectrum. Profiling of secondary metabolites from a species could be assessed to understand the therapeutic potential of these compounds. This will also
Dr. S. K. M. Habeeb

Department of Genetic Engineering, School of Bioengineering, SRM Institute of Science and Technology (formerly SRM University), Kattankulathur, Near Chennai - India. Email: habeebm@ srmist.edu.in

open up an interesting approach of target identification, probably from the genomic data. Differential expression studies are proven to be one of the very good methods for the identification of molecular markers, gene and protein targets. Computer aided drug discovery approaches involves molecular modeling of the identified protein target, structural profiling of the target protein binding site, high-throughput molecular docking and virtual screening to shortlist potential lead compounds and finally, thermodynamic features-based monitoring of the protein-lead complex motion using the simulation strategies.

The need of the hour for bioinformatics and computational biology is to come up with novel computer software tools that can perform accurate predictions with greater efficiency. Emergence of artificial intelligence has become one of the go-to technologies for the deep understanding of the biological phenomenon such as protein-protein interactions, biological pathway maps, data mining and most importantly simulation of these biological data to design predictive approaches leading to welfare of mankind.

Through the ICIBCoB conference we aim to unite experimental and in-silico biologist under one forum. ICIBCoB will act as a platform to discuss and deliberate on the new software tools and databases to be developed, need for novel algorithms for solving and understanding genome-metabolome relationship from the omics data, avenues for the augmentation of the CADD methods and design quick data mining and annotation strategies. ICIBCoB will give the academic researchers to brainstorm on the needs of the industry requirements, thereby, producing skilled bioinformatics professionals. 